\title{
The Effect of Positioning on Adaptation to Spontaneous Breathing in Premature Infants After Weaning from Mechanical Ventilation: A Randomized Controlled Trial
}

\author{
國Funda Güler ${ }^{1}$, 回Hüsniye Çalışır² \\ 1Aydın Adnan Menderes University, Application and Research Hospital, Neonatal Intensive Care Unit, Aydın, Turkey \\ ${ }^{2}$ Aydın Adnan Menderes University Faculty of Nursing, Department of Pediatric Nursing, Aydın, Turkey
}

\begin{abstract}
Aim: To determine the effects of positioning on the adaptation to spontaneous breathing in premature infants after weaning from mechanical ventilation.

Materials and Methods: This randomized controlled experimental study was conducted with 60 (study group=30; control group=30) premature infants in the neonatal intensive care unit. The infants in the study group and the control group were in a prone position and a supine position, respectively, during the first 120 minutes when spontaneous breathing started after they were weaned from mechanical ventilation.

Results: There were significant intragroup differences in mean heart rate between repeated measurements at different time points. The differences were not found to be due to positioning. The mean $\mathrm{SpO}_{2}$ was higher in the study group than in the control group, although the difference was not significant. This finding suggested that positioning did not have an influence on $\mathrm{SpO}_{2}$. Two infants in the control group failed to maintain spontaneous breathing. Although the study group had a significantly higher respiratory rate than the control group, the difference was not significant. Therefore, positioning was not found to affect respiratory rate.
\end{abstract}

Conclusion: There was no effect of positioning on the adaptation to spontaneous breathing in premature infants after weaning from mechanical ventilation.

Keywords: Mechanical ventilation, premature infants, prone position, supine position, breathing

\section{Introduction}

Appropriately positioning infants in the neonatal intensive care unit (NICU) is an important practice that helps with neuromuscular maturation of premature infants, regulates sleep patterns, alleviates nutritional problems, and reduces pain and stress (1-8). Positioning neonates in a suitable manner may aid infants in developing posture and mobility; conversely, failing to appropriately position infants may lead to short- and long-term posture-related developmental issues (9).

Whether to place infants in the supine and/or prone position is a matter that has been much discussed in the current literature. It has been shown that the prone position is not safe for healthy infants born at term and that it 
increases the risk of sudden infant death (10-12). Since 1992, the recommendation has been to place infants under one year of age in the supine position to reduce sudden Infant Death syndrome (13). In contrast, other studies have shown that, in the hospital setting, placing premature infants in the prone position yields benefits $(6,9,14-17)$.

The prone position may contribute to increased respiratory functions, especially oxygenation, by reducing expired $\mathrm{CO}_{2}$, increasing diaphragm compliance and function, and increasing thoracic-abdominal respiratory synchronization $(9,14,17)$. One study reported that placing a ventilated premature infant in a prone position had a positive effect on oxygenation. The same study also revealed that preterm infants placed in the prone position experienced greater oxygen saturation, had fewer events of desaturation, cried less than infants left in a supine position, displayed fewer stress responses and displayed quieter sleep patterns (6). Another study reported that infants placed in the prone position showed an increase in $\mathrm{PaO}_{2}$ levels and drops in end-tidal $\mathrm{PaCO}_{2}$ and respiratory rate (RR) (9). It was asserted in one study that placing premature infants on nasal continuous positive airway pressure (CPAP) on their left sides or in a prone position increases oxygen saturation and tidal volume and reduces $\mathrm{PaCO}_{2}$ (15).

In a study probing the association between cardiorespiratory stability and positioning in premature infants with symptomatic apnea, it was discovered that no difference existed between prone and supine positioning in terms of apnea, bradycardia or desaturation episodes, but that the incidence of total oxygen desaturation was higher in the supine position (18).

Various other studies have pointed to the effects of positioning premature infants while they are under mechanical ventilation (MV) and during the time they are being weaned from MV $(14,19,20)$. However, few studies were found in the literature about the effects of positioning a baby after weaning from MV (21).

This study was conducted to examine the effect of positioning premature infants in two different positions (supine and prone) on their adaptation to spontaneous respiration after being weaned from $\mathrm{MV}$.

\section{Materials and Methods}

This randomized controlled experimental study was carried out at the NICU of a university hospital in Turkey. Infants in the NICU are routinely and frequently placed in a supine position or on their left or right sides but rarely placed in a prone position. The criteria for inclusion in the sample of premature infants were as follows: being born at gestational weeks 30-36, having no congenital abnormalities, the absence of intracranial bleeding and/or periventricular leukomalacia, and not having undergone any surgical interventions.

The sample size was determined according to arterial oxygen saturation $\left(\mathrm{SpO}_{2}\right)$ means and standard deviations by a power analysis with a pilot implementation with four premature infants after they were weaned from MV in the NICU. The analysis resulted in an effect size $(d)=0.22$ and $\alpha=0.05$ at a confidence interval of $95 \%$ and power $=0.91$; the number of premature infants needed for the sample was thus.

The premature infants included in the study were separated into study and control groups using simple randomization. A total of 60 premature infants matching the recruitment criteria were included in the study: 30 in the study group (prone position) and 30 in the control group (supine position) (Figure 1).

The data were collected with a neonatal data collection form that was designed based on examples in the literature $(14,18)$. Furthermore, some of the measured data used in the study were obtained from the mechanical ventilator and the bedside patient monitors used in the NICU.

The neonatal data collection form included 27 items querying the sociodemographic characteristics of the infants, their MV parameters, their $\mathrm{SpO}_{2}$ levels, their vital signs, the presence of complications and the use of medications.

The mechanical ventilator used in this study was a calibrated, conventional infant ventilator with a highfrequency oscillator. The bedside patient monitors used in the NICU in which the study was conducted were calibrated devices of different brands and models with the same specifications and functions and also had the ability to be used with neonatal patients. These devices measured the parameters of ECG/heart rate (HR), oxygen saturation, temperature, noninvasive blood pressure and respiration count.

The final parameters from the ventilated, premature infants in the study were recorded. Following this, the infants were separated into the study and control groups and positioned. After removal from the ventilator, the infants in the study group were placed in a prone position, and the infants in the control group were placed in a supine position. Oxygen support was continued during the procedure. In both groups, the infants were provided free $\mathrm{O}_{2}$ support as the standard protocol of the unit. In the first 120 


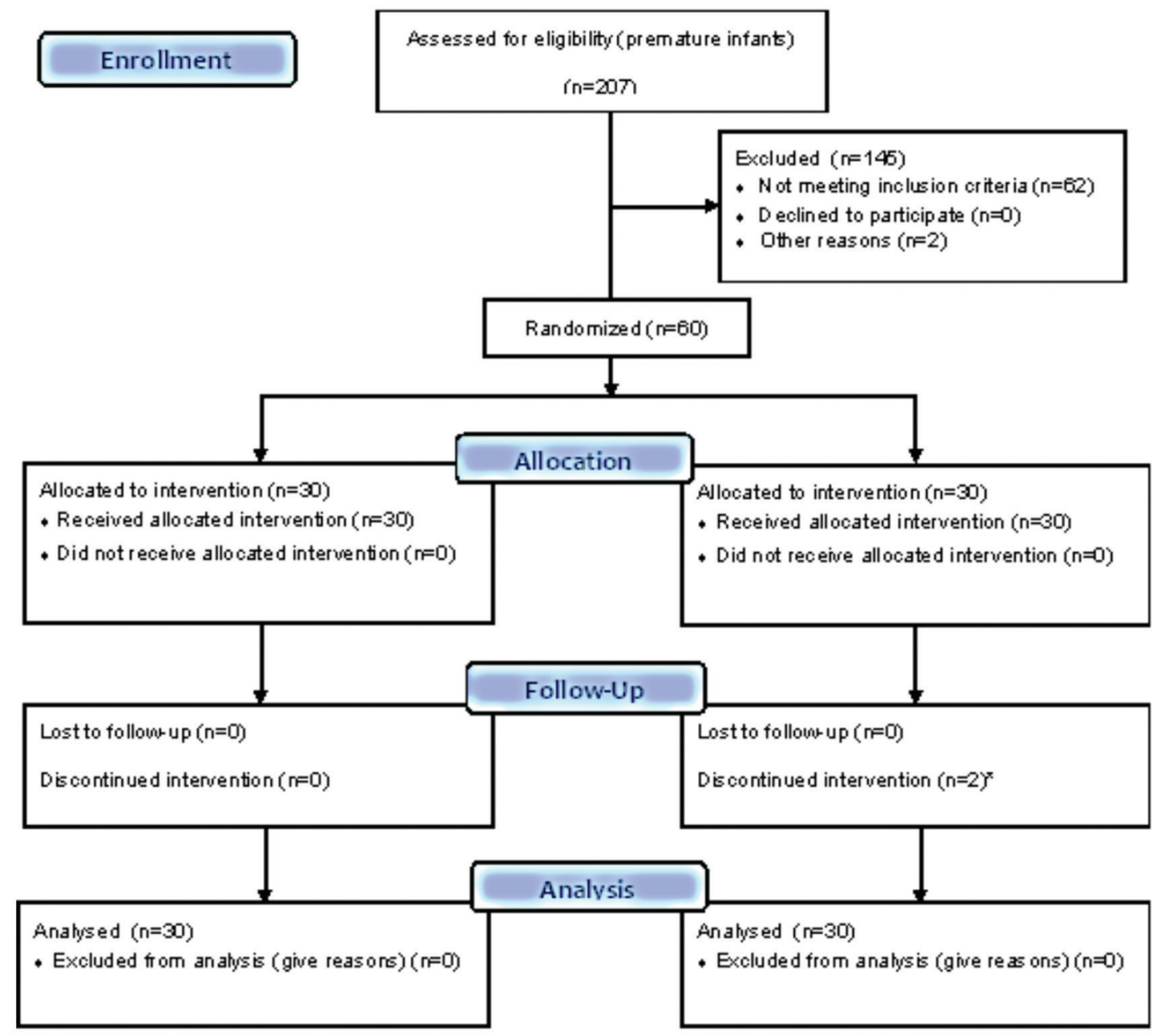

"Two infants in the control group (at the 60th and 100th minutes) were returned to the mechanical ventilator by the neonatal intensive care team due to ume kened respir atony strength, continuation of intercostal retractions, continued superficial and tachypneic respiration ( $60 / \mathrm{min})$. development of apnea, symptoms of ainway obstruction and an increased need for oxygen. Therefore, the followsup result and the repeated measures were not anabyed for one baby after the 60th minute and for a second baby after the 100th minute.

Figure 1. CONSORT Flow Diagram

minutes after the infants were removed from MV, they were evaluated in terms of $\mathrm{HR}, \mathrm{SpO}_{2}$ and RR every 20 minutes. The infants were also monitored in terms of breathing difficulties and the presence of apnea.

Data were evaluated with descriptive statistics, the Kolmogorov-Smirnov test, chi-square test and Yates' Continuity Correction chi-square test, Student's t-test, Mann-Whitney $U$ test and analysis of variance.

\section{Results}

The study and control groups were homogeneous in terms of gestational age, and weight. The postnatal ages of the premature infants in the study group were older than the infants in the control group (Table I).
The groups were homogeneous in terms of respiratory distress syndrome, transient tachypnea of the newborn, atelectasis, patent ductus arteriosus and the presence of maternal gestational diabetes. Moreover, the groups were similar in terms of their positioning before being weaned from the mechanical ventilator, and the ventilator mode.

The rate of caffeine use among the babies enrolled in the study group was found to be $50 \%(n=15)$ and that of the control group was $40 \%(n=12)$. According to this, it was determined that the rates of caffeine use in the study and control groups were similar $(p>0.05)$.

It was found that prior to monitoring, the groups exhibited differences in RR and mean systolic blood pressure 
(SBP); however, MV parameters, other vital signs and blood gas values were homogeneous. The mean RR of the infants in the study group was lower than the mean RR of the infants in the control group. However, the mean SBP of the infants in the study group was higher than the mean SBP of the infants in the control group (Table I).

Statistically significant differences were found in the repeated measurements of HR in all of the premature infants in this study $(p=0.003)$. The position of the infants was not found to have an effect on the differences in HR in the study and control groups ( $p=0.492$; Figure 2 ).

Statistically significant differences were found in the repeated saturation measurements in all of the premature infants in the study $(p<0.001)$. Position, however, was not found to have an impact on the saturation values of the premature infants ( $p=0.717$; Figure 3 ).

There were no differences among the repeated measurements of RR for the infants in the study group $(p=0.637)$, and in the control group $(p=0.750)$. Furthermore, no statistically significant difference could be found between the two groups in terms of mean RR ( $p=0.882$; Figure 4$)$.

Examining whether there were any differences between the premature infants in the study and control groups in terms of the presence of respiratory distress, no statistically significant differences were seen between the groups at minute $0,20,40,60,80,100$ or 120 of monitoring. It was thus observed that the respiratory distress findings for the two groups during the monitoring period were similar. On the other hand, while there was no development of apnea in any of the infants in the study group over the course of the monitoring period, one infant in the control group was

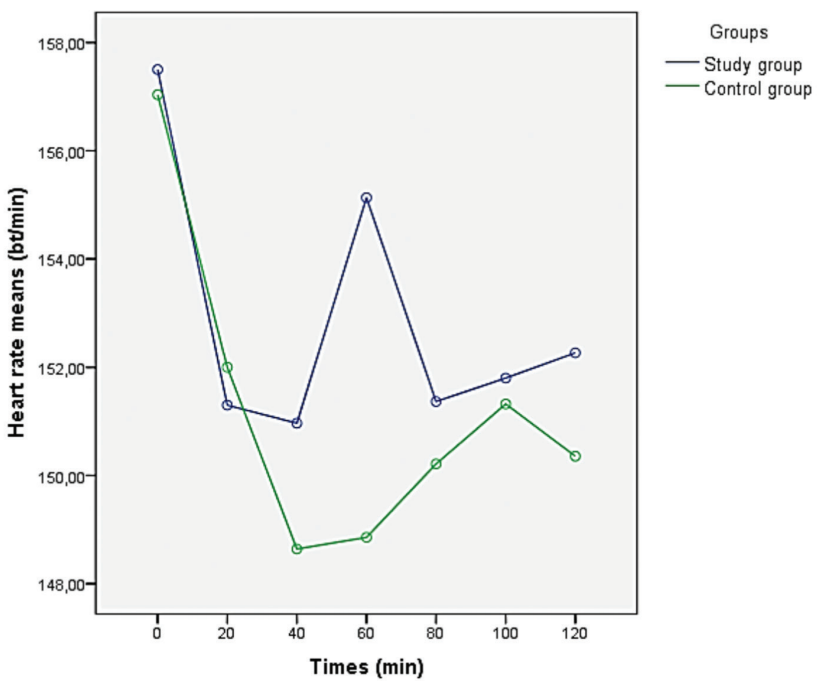

Figure 2. Distribution of mean heart rates over the monitoring period in the study and control groups

Table I. Post-natal characteristics, MV parameters, vital signs and blood gas values prior to weaning from mechanical ventilator $(n=60)$

\begin{tabular}{|c|c|c|c|}
\hline Variables & Study Group $(n=30)$ Mean \pm SD & Control Group $(n=30)$ Mean \pm SD & Test; $\mathbf{p}$ value \\
\hline Gestational age (weeks) & $31.83 \pm 2.19$ & $32.16 \pm 1.89$ & $t=-0.629 ; p=0.532$ \\
\hline Birth weight (gr) & $1,612.60 \pm 670.56$ & $1,681.50 \pm 534.65$ & $t=-0.440 ; p=0.662$ \\
\hline Current weight (gr) & $1,639.40 \pm 619.90$ & $1,634.06 \pm 522.40$ & $t=0.036 ; p=0.971$ \\
\hline Age (days) & Median (min-max) 4.00 (0-98) & Median (min-max) $2.00(0-26)$ & $z=-2.517 ; p=0.012$ \\
\hline $\mathrm{CPAP}\left(\mathrm{cm} \mathrm{H}_{2} \mathrm{O}\right)$ & $6.53 \pm 0.51$ & $6.16 \pm 0.71$ & $t=1.544 ; p=0.135$ \\
\hline $\operatorname{PEEP}\left(\mathrm{cm} \mathrm{H}_{2} \mathrm{O}\right)$ & $5.53 \pm 0.83$ & $5.66 \pm 0.84$ & $t=-0.455 ; p=0.652$ \\
\hline $\mathrm{PIP}\left(\mathrm{cm} \mathrm{H}_{2} \mathrm{O}\right)$ & $20.73 \pm 3.16$ & $20.93 \pm 3.61$ & $t=-0.228 ; p=0.820$ \\
\hline $\mathrm{FiO}_{2}(\%)$ & $25.10 \pm 7.42$ & $24.66 \pm 8.09$ & $t=0.216 ; p=0.830$ \\
\hline Rate (respiration/min.) & $31.33 \pm 10.43$ & $32.44 \pm 12.12$ & $t=-0.279 ; p=0.782$ \\
\hline Body temperature $\left({ }^{\circ} \mathrm{C}\right)$ & $36.53 \pm 0.29$ & $36.50 \pm 0.24$ & $t=0.475 ; p=0.636$ \\
\hline Respiration rate (/min.) & $57.26 \pm 6.52$ & $62.03 \pm 9.95$ & $t=-2.193 ; p=0.032$ \\
\hline Heart rate (/min.) & $153.33 \pm 14.00$ & $150.50 \pm 17.06$ & $t=0.703 ; p=0.485$ \\
\hline Systolic blood pressure $(\mathrm{mmHg})$ & $71.93 \pm 14.36$ & $64.36 \pm 11.07$ & $t=2.285 ; p=0.026$ \\
\hline Diastolic blood pressure $(\mathrm{mmHg})$ & $38.40 \pm 10.94$ & $35.76 \pm 8.89$ & $t=1.023 ; p=0.311$ \\
\hline $\mathrm{SpO}_{2}(\%)$ & $96.20 \pm 2.69$ & $95.36 \pm 3.01$ & $t=1.129 ; p=0.263$ \\
\hline $\mathrm{pH}$ & $7.35 \pm 0.04$ & $7.36 \pm 0.05$ & $t=-0.486 ; p=0.636$ \\
\hline $\mathrm{pCO}_{2}(\mathrm{mmHg})$ & $39.02 \pm 4.85$ & $37.18 \pm 10.81$ & $\mathrm{t}=0.322 ; \mathrm{p}=0.753$ \\
\hline
\end{tabular}

t: Student's t-test; SD: Standard deviation, CPAP: Continuous positive airway pressure, Min: Minimum, Max: Maximum, PEEP: Positive end-expiratory pressure 


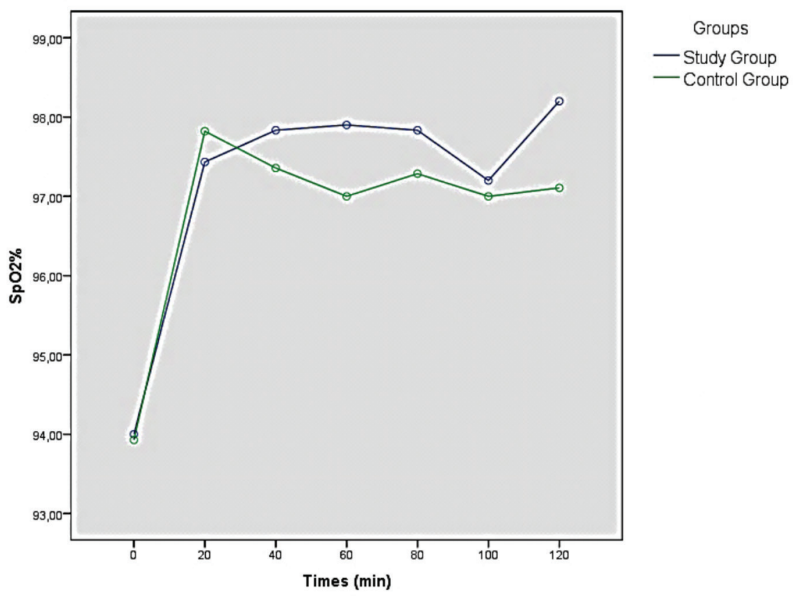

Figure 3. Distribution of mean saturation levels $\left(\mathrm{SpO}_{2}\right)$ over the monitoring period in the study and control groups

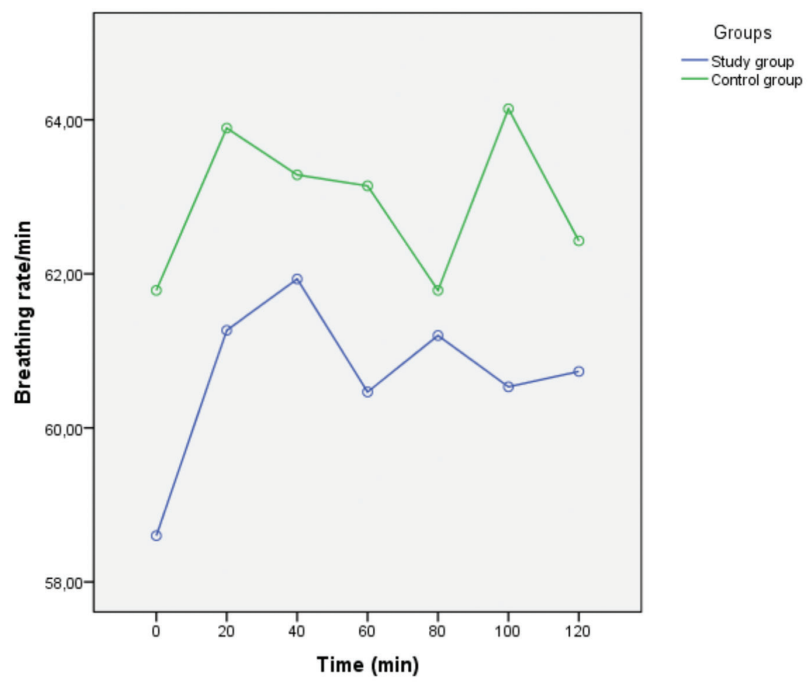

Figure 4. Distribution of mean respiration rates over the monitoring period in the study and control groups

seen to develop apnea twice. None of the infants in the study or control group were re-intubated. However, noninvasive MV was administered to two infants in the control group (but none in the study group) because they could not keep up spontaneous respiration. None of the infants was found to have developed atelectasis during follow-up after extubation.

\section{Discussion}

In this study, we found no difference between placing premature infants in a prone or supine position for any of the parameters of $\mathrm{RR}, \mathrm{SpO}_{2}$ value or respiratory distress in the repeated measurements taken after the infants were weaned from the MV. In addition, placing the infants in a different position did not have an impact on their change in HR.
The mean $\mathrm{SpO}_{2}$ value of the study group throughout monitoring was higher than in the control group, but this difference was not found to be statistically significant. This finding shows that position has no effect on mean $\mathrm{SpO}_{2}$ levels. The fact that there were no infants in the study group that were returned to the ventilator but that there were two infants in the control group that were reconnected to the ventilator may be interpreted as evidence in favor of prone positioning. The prone position may contribute to improved respiratory function, especially oxygenation, by reducing expired $\mathrm{CO}_{2}$, increasing diaphragm compliance and function and increasing thoracic-abdominal respiratory synchronization $(9,14)$. In one study, intubated infants at gestational weeks 25-36 were monitored postnatally for a week and their positions were changed every 2 hours. Those infants lying in a prone position were found to display less motor activity, higher oxygen saturation and less desaturation than those infants lying in a supine position (6). Another study with premature infants using CPAP reported a significant difference between the mean saturation levels of infants in a prone position and those of infants lying on their right or left sides; it was observed that infants in a prone position exhibited the highest saturation levels (22). A study exploring the effect of positioning on the successful weaning of premature infants from a mechanical ventilator did not find a significant difference in mean $\mathrm{SpO}_{2}$ levels between the two groups of infants in the study who were lying in supine and prone positions (14). In a systematic review, Balaguer et al. (19) reported an increase in $\mathrm{PaO}_{2}$ in the prone position compared to the supine position, as well as a slight improvement in oxygen saturation. The same study showed a slight reduction in the number of desaturation episodes in the prone position. Rivas-Fernandez et al. (23) found an increase in arterial oxygen tension $\left(\mathrm{PO}_{2}\right)$ and $\mathrm{SpO}_{2}$, and also noted a slight improvement in the number of episodes of desaturation in the prone position compared with the supine position. In a study exploring the association between cardiorespiratory stability and positioning in premature infants with symptomatic apnea, it was discovered that no difference existed between infants in the supine and prone position in terms of apnea and desaturation episodes, but that the incidence of total oxygen desaturation was higher in the supine position (18). Studies researching the correlation between the positioning of premature infants and oxygenation have been largely devoted to infants connected to a ventilator. The different result in the present study, revealing no difference in saturation levels between infants lying in the prone and supine positions, may have stemmed from the differences in the sample group and study methods. 
While no infant in the study group exhibited apnea, the appearance of two cases of apnea episodes in the control group may be considered an important finding that is consistent with the literature. A review of the literature reveals that the prone position is recommended for effective respiration, $\mathrm{SpO}_{2}$ and the baby's stabilization; it has been reported that the prone position reduces apnea in premature infants $(9,22,24)$. In another study, it was found that there were no differences between the prone and supine positions in terms of the incidence of apnea or the longest duration of apnea (18).

While the RRs of both groups were close to each other at the $80^{\text {th }}$ minute, the mean RRs measured over all the monitoring period were lower in the infants in the control group compared to those in the study group. While the RR of the study group throughout the monitoring period was lower than in the control group, this difference was not found to be statistically significant. Accordingly, it was shown that position did not influence the RR.

Other studies that explored the effect of the position of a premature infant on the RR, symptoms of respiratory distress and the existence of apnea reached different conclusions $(9,14,22)$. In a study of premature infants on CPAP, no difference was found in the RR of infants lying in a supine position, a prone position or on their right or left sides (22). In a study in which the effect of positioning premature infants on $\mathrm{MV}$ on the success of weaning from MV was examined, no difference was observed between the supine and prone positions in terms of the infant's RRs (14). Another study reported that infants placed in the prone position experienced a diminished RR. It has also been reported that the prone position contributes to chest wall synchronization and an improvement in respiration (9). The reason that the RR of the infants in the study and control groups was different from the research results may stem from the differences in the sample group and study methods.

Periodic respiration is frequently observed in premature infants. Superficial respiration, tachypnea, bradypnea, apnea, unequal respiratory sounds and retracting are signs of respiratory distress. In evaluating premature infants in a study on respiratory distress, the symptoms that were examined were retractions, collapse of the thorax due to difficulty breathing, superficial respiration and breathing through the nostrils. No differences were found between the two groups of premature infants in the study in terms of respiratory distress in the measurements taken throughout the monitoring period. In other words, the position of infants after being weaned from MV was not found to have an impact on the symptoms of respiratory distress. On the other hand, the fact that the infants in the study in both prone and supine positions exhibited similarities in terms of the existence of respiratory distress and that some of the measurements displayed the same distribution may be considered significant findings of the study.

The premature infants in the study group were observed to determine whether there were any differences in their HRs over the course of the monitoring period. After being weaned from $M V$, the $H R$ at minute 0 revealed a strikingly higher value in both groups compared to their HR measured at minute 20. This finding may be considered important in that it provides insight into the way infants weaned from MV respond to the stress they are exposed to immediately after weaning and insight into how this stress affects their vital signs. The intragroup HRs of all of the infants in the study group were different across the repeated measurements. However, no impact of position on HR was found. In the review of the literature, very few studies were found that explored the relationship between positioning premature infants and their HRs $(14,18,22)$. In a study of premature infants on CPAP, no differences were found in the HR of infants lying in a supine position, a prone position or on their right or left sides (22). In a study probing the association between cardiorespiratory stability and positioning in premature infants, it was discovered that no difference existed between the prone and supine positions in terms of bradycardia episodes (18). In a study in which the effects of positioning premature infants on $M V$ on the success of weaning from MV was examined, no differences were observed in HR between the supine and prone position groups (14)

\section{Study Limitations}

There were some limitations in this study. First, during MV in the NICU where the study occurred, there was no standardization in the weaning period or later in feeding times, and this issue may have led to possible effects on the infants' respiration even though feeding was handled orogastrically according to appropriate procedures. The second factor was that because the weaning from MV occurred at different times, there were differences in the sleep-wake cycles among the infants, and these differences may have affected cardiorespiratory responses $(15,21)$. The third limitation was the fact that the non-homogeneity of the infants in the two groups in terms of postnatal age, respiration and SBP values prior to MV may have had an impact on the results. Despite these limitations, however, 
the study is of worth in our country as it is the first study to determine the impact of positioning premature infants on spontaneous respiration after weaning from MV.

\section{Conclusion}

This study did not find any differences in the effect produced by placing premature infants in a prone or supine position on any of the parameters of RR and respiratory distress in repeated measurements taken after the infants were weaned from MV. There were differences in HRs and $\mathrm{SpO}_{2}$ levels in the repeated measurements in all of the infants after they were weaned from MV. However, placing the infants in a different position did not have an impact on their changes in $\mathrm{HR}$ and $\mathrm{SpO}_{2}$ levels. While none of the infants in the study group developed apnea, apnea developed twice in one of the infants in the control group.

Acknowledgements: The authors thank the premature infants and their parents who agreed to participate in this study for their contribution.

\section{Ethics}

Ethics Committee Approval: Permission for this study was obtained from the chief physician of the hospital. Ethical approval of the study was granted by the ethical committee for clinical research (approval no. 2012-153).

Informed Consent: Written informed consent was obtained from the parents before the premature newborns participated in the study.

Peer-review: Externally and internally peer-reviewed.

\section{Authorship Contributions}

Study Design: H.Ç., F.G., Data Collection: F.G., Analysis and Interpretation: H.Ç., F.G., Literature Search: H.Ç., F.G., Writing: F.G., H.Ç.

Conflict of Interest: No conflict of interest was declared by the authors.

Financial Disclosure: The authors declared that this study received no financial support.

\section{References}

1. Keller A, Arbel N, Merlob P, Davidson S. Neurobehavioral and autonomic effects of hammock positioning in infants with very low birth weight. Pediatr Phys Ther 2003;15:3-7.

2. Bhat RY, Hannam S, Pressler R, Rafferty GF, Peacock JL,Greenough A. Effect of prone and supine position on sleep, apneas, and arousal in preterm infants. Pediatrics 2006;118:101-7.

3. Park J, Thoyre S, Knafl G), Hodges EA, Nix WB. Efficacy of semi elevated side-lying positioning during bottle-feeding of very preterm infants: a pilot study. I Perinat Neonatal Nurs 2014;28:69-79.
4. Alinejad-Naeini $M$, Mohagheghi $P$, Peyrovi $H$, Mehran A. The effect of facilitated tucking during endotracheal suctioning on procedural pain in preterm neonates: a randomized controlled crossover study. Glob J Health Sci 2014;6:278-84.

5. Cândia MF, Osaku EF, Leite $M A$, et al. Influence of prone positioning on premature newborn infant stress assessed by means of salivary cortisol measurement: pilot study. Rev Bras Ter Intensiva 2014;26:169-75.

6. Chang YJ, Anderson GC, Lin CH. Effects of prone and supine positions on sleep state and stress responses in mechanically ventilated preterm infants during the first postnatal week. I Adv Nurs 2002;40:161-9.

7. Grenier IR, Bigsby R, Vergara ER, Lester BM. Comparison of motor self-regulatory and stress behaviors of preterm infants across body positions. Am J Occup Ther 2003;57:289-97.

8. Peng NH, Chen LL, Li TC, Smith M, Chang YS, Huang LC. The effect of positioning on preterm infants' sleep-wake states and stress behaviors during exposure to environmental stressors. I Child Health Care 2014;18:314-25.

9. Monterosso L, Kristjanson L, Cole J. Neuromotor development and the physiologic effects of positioning in very low birth weight infants. J Obstet Gynecol Neonatal Nurs 2002;31:138-46.

10. Blair PS, Sidebotham P, Berry PJ, Evans M, Fleming PJ. Major epidemiological changes in sudden infant death syndrome: $A$ 20-year population-based study in the UK. Lancet 2006;367:3149.

11. Li DK, Petitti DB, Willinger $M$, et al. Infant sleeping position and the risk of sudden infant death syndrome in California, 1997-2000. Am J Epidemiol 2003;157:446-55.

12. Task Force on Sudden Infant Death Syndrome, Moon RY. SIDS and other sleep-related infant deaths: expansion of recommendations for a safe infant sleeping environment. Pediatrics 2011;128:e1341-67.

13. American Academy of Pediatrics. Changing concepts of sudden infant death syndrome: implications for infant sleeping environment and sleep position. Task force on infant sleep position and sudden infant death syndrome. Pediatrics 2000;105:650-6.

14. Antunes LC, Rugolo LM, Crocci AJ. Effect of preterm infant position on weaning from mechanical ventilation. ) Pediatr (Rio J) $2003 ; 7: 239-44$

15. Gouna G, Rakza T, Kuissi E, Pennaforte T, Mur S, Storme L. Positioning effects on lung function and breathing pattern in premature newborns. J Pediatr 2013;162:1133-7.

16. van der Burg PS, Miedema $M$, de Jongh $F H$, Frerichs I, van Kaam $\mathrm{AH}$. Changes in lung volume and ventilation following transition from invasive to noninvasive respiratory support and prone positioning in preterm infants. Pediatr Res 2015;77:484-7.

17. Wu J, Zhai J, Jiang $\mathrm{H}$, et al. Effect of change of mechanical ventilation position on the treatment of neonatal respiratory failure. Cell Biochem Biophys 2015;72:845-9.

18. Keene DJ, Wimmer JE Jr, Mathew OP. Does supine positioning increase apnea, bradycardia and desaturation in preterm infants? J Perinatol 2000;20:17-20. 
19. Balaguer A, Escribano J, Roqué i Figuls M, Rivas-Fernandez M. Infant position in neonates receiving mechanical ventilation. Cochrane Database Syst Rev 2013 Mar 28;CD003668.

20. Sud S, Sud M, Friedrich JO, Adhikari NK. Effect of mechanical ventilation in the prone position on clinical outcomes in patients with acute hypoxemic respiratory failure: A systematic review and meta-analysis. CMAI 2008;178:1153-61.

21. Elder DE, Campbell A), Galletly D. Effect of position on oxygen saturation and requirements in convalescent preterm infants. Acta Pediatr 2011;100:661-5.
22. Brunherotti MA, Martinez EZ, Martinez FE. Effect of body position on preterm newborns receiving continuous positive airway pressure. Acta Paediatr 2013;103:101-5.

23. Rivas-Fernandez M, Roqué i Figuls M, Diez-Izquierdo A, Escribano J, Balaguer A. Infant position in neonates receiving mechanical ventilation. Cochrane Database Syst Rev 2016;CD003668.

24. Zhao J, Gonzalez F, Mu D. Apnea of prematurity: from cause to treatment. Eur J Pediatr 2011;170:1097-105. 